\title{
Lumbo-Pelvic and Lower Limb Joint Kinematics in Subjects with and Without Low Back Pain: A Protocol for Systematic Review
}

\section{Fatemeh Ghasemi Dehcheshme}

University of Social Welfare and Rehabilitation Science

Massoud Arab ( $\square$ arabloo_masoud@hotmail.com )

University of social walfare and rehabilitation sciences https://orcid.org/0000-0003-2371-6100

Mohammad Reza Nourbakhsh

University of North Georgia

\section{Protocol}

Keywords: low back pain, systematic review, kinematics, lumbo-pelvic, lower extremity

Posted Date: December 20th, 2021

DOI: https://doi.org/10.21203/rs.3.rs-1164346/v1

License: (c) (1) This work is licensed under a Creative Commons Attribution 4.0 International License.

Read Full License 


\section{Abstract \\ Background}

Low back pain (LBP) is one of the leading causes of disability worldwide. While its main cause is mostly unknown, more than $80 \%$ of people experience this disorder during their lifetime. Some clinical evidence suggests that people with chronic LBP have various lower extremity movement disorders. This study aims to systematically review the literature on comparing the kinematics of the lumbopelvic region and lower limb joints in people with non-specific low back pain (NS-LBP) compared to healthy individuals.

\section{Methods/design:}

We will search main electronic databases (Medline/PubMed, Scopus, Embase, Web of Science, Pedro, ProQuest and Google Scholar) and two key journals (Journal of Electromyography and Kinesiology, Clinical Biomechanics). Also, the reference list of the previous systematic reviews would be handsearched. Studies that have examined the kinematic differences in lumbopelvic or lower limbs between NS-LBP and healthy individuals will be included. We will examine the methodological quality and statistical reporting of each eligible trial using the Newcastle - Ottawa Scale (NOS).

\section{Discussion}

To our knowledge, this study will be the first systematic review to examine the kinematic differences of the lower limb joints and pelvic complex between healthy people and NS-LBP. Since the kinematic assessment of the lower extremities and pelvic joints during daily activities may help understand underlying factors causing LBP, it is important to evaluate the kinematic changes in these regions, during repetitive daily functions in these patients.

\section{Systematic review registration:}

PROSPERO (CRD42021230826)

\section{Background}

Currently, Low back pain (LBP) is one of the most common neuromuscular disorders that causes occupational and social disabilities, poor quality of life, emotional problems, and absenteeism [1-4]. LBP that lasts for more than three months is called chronic low back pain (CLBP) [5]. However, in a large percentage of people with LBP, people recover within nine months to a year after the onset of the disorder [6]. Since the lower limb-pelvic set is an important motor chain for the spine [7], studying the kinematic properties of the components of this kinematic chain may provide useful evidence. Some researchers 
suggested an association between LBP and impaired lumbar-pelvic girdle control, with an increased risk of lower limb injuries [8-10].

People with LBP may suffer from hip disorders, defects in the range of motion (ROM), and reduced strength in one or both thighs [11-16]. Although the findings of some studies indicate an association between LBP and the hip joint, the nature of these relationships is not fully understood [17]. Also, two studies that have evaluated the kinematics of the lower extremities in people with LBP, confirm the kinematic changes of the knee joint in people with LBP compared to healthy people $[18,19]$.

Abnormal position and function of the foot has also been suggested as a possible risk factor for LBP [20]. In previous studies, LBP patients showed differences in the performance of functional tasks when compared to healthy individuals; For example, some cases showed a decrease in pelvic and lumbar movement during activity, which may indicate a change in pelvic kinematics [21]. Therefore, there is some evidence to support an association between lower extremity and pelvic joint disorders and LBP. Most previous studies have examined and compared the kinematics of the lumbar spine region in healthy people with LBP, and few studies have examined the pelvic lumbar region and lower limb joints in these individuals. Some review studies have examined the kinematic differences between healthy and LBP individuals [22, 23]. In [22], Laird and colleagues investigated the kinematic differences of the lumbopelvic region in different functions, between LBP and healthy individuals. They showed a decrease in the proprioception and ROM in the lumbar region, as well as a slower execution of movements in people with LBP relative to healthy individuals. In the review conducted by laird et al., studies performed on people with different types of LBP are also included [22].

LBP is a multidimensional disorder that various factors contribute to its development $[24,25]$. Therefore, providing a classification that contains various studies is important to make the treatment more effective [26]. Nearly $85-90 \%$ of patients with LBP are classified in non-specific low back pain (NS-LBP) group without specific pathology such as disc herniation [27, 28]; therefore, in this review, we will include only studies performed on NS-LBP cases. Also, in the present study, in addition to the kinematic parameters of the lumbopelvic region, which were examined in [22], studies comparing kinematic parameters in the lower extremities of NS-LBP with healthy participants will also be included.

In another review of lifting performance, people with LBP showed slower movement and greater stiffness in the lower back, as well as a greater range of knee flexion than healthy people [23]. In their review, all studies on lifting function in different groups of LBP have been reviewed, while in the present study, in addition to lifting function, we have also included studies on different functions only in the NS-LBP group.

In a review study on the relationship between hip joint ROM and NS-LBP, the results showed that ROM of internal rotation in the hip joint was limited in patients with NS-LBP than in healthy individuals [29]. In their review, only the relationship between the ROM of the hip joint in different situations in people with NS-LBP compared to healthy people has been investigated. Contrary to the review presented in [29], 
studies on the lumbopelvic region and other lower limb joints or studies that examined other kinematic parameters than ROM will be included in the study.

To the best of our knowledge, a systematic review study has not yet examined the kinematic differences between lumbopelvic and lower limb joints between NS-LBP and healthy people. Observation and analysis of movement quality in patients with NS-LBP is important for physiotherapists to make clinical decisions and exercise program design [30].

The results of this study may help to determine clinically in patients with NS-LBP, which of the kinematic parameters should be more focused on the lumbopelvic region or lower limb joints; or determine, which kinematic variables and functions can be helpful in detecting people with NS-LBP. Our aim is to conduct a systematic review study on the kinematic differences of the pelvic and lower limb joints during the performance of different functions in NS-LBP compared to healthy individuals.

\section{Methods/design}

This protocol was developed based on the Preferred Reporting Items for Systematic Reviews and MetaAnalyses Protocol (PRISMA-P) checklist (see additional file 1) [31]. Also, it has been registered with the International Prospective Register of Systematic Reviews (PROSPERO) database (CRD42021230826).

\section{Population}

Due to the high heterogeneity in the mechanisms involved in the occurrence of LBP and to prevent heterogeneity in the studies that will be included in this review, in terms of the mechanisms that cause LBP in people participating in these studies, only people with NS-LBP without any neurological symptoms shooting into the lower extremities will be included. Inclusion criteria for studies based on participants include: age range of 18 to 60 years old, absence of any history of orthopedic surgery in the lumbar-pelvic area or lower extremities, absence of deformity in the vertebral area or lower extremity, absence of any neurological and vestibular disorders, absence of shooting pain or nerve symptoms in the lower extremities. Just analytical observational studies that have investigated one or both sexes and provided a comparison of kinematic variables in people with NS-LBP with healthy individuals will be included. To have a study base in comparing the two groups of LBP and healthy, studies conducted on only one group of healthy people or LBP will not be included in the study.

\section{Exposure:}

Differences in kinematic parameters such as joint ROM, segment alignment, angular displacement, joint angle, average velocity (AV), maximal velocity (MV), maximal acceleration, lumbar/hip motion ratios (L/H ratio), kinematic symmetry, and other kinematic parameters that have been compared between people with NS-LBP and healthy in studies under the same conditions.

In this review study, part of our focus is on studies that have examined the area of lumbar movements relative to the pelvis. In walking function compared to most daily functions, the lumbar region has less 
movement relative to the pelvis and lower limbs, it seems that Gait performance, unlike most daily functions that required end-range spinal movement, does not determine the kinematic differences between low back pain and healthy individuals. Also, walking does not provocative for the most subjects with LBP [32]. Furthermore, more previous studies have focused on gait, we will not include studies on gait in the review.

Studies that have collected data using the various motion capture systems, including mechanical systems, magnetic systems, inertial systems, and optical systems, will be included in this review study.

\section{Outcome:}

In this review, studies comparing NS-LBP (acute, sub-acute, or chronic) with healthy individuals will be included. NS-LBP is the pain felt between the lower ribs and the gluteal fold, and for which no specific pathophysiological cause can be determined [33].

\section{Study designs/characteristics:}

Full text of eligible analytical observational studies (case-control, cross sectional and cohort) available in English intended to investigate the kinematic parameters in NS-LBP patients in comparison with healthy individuals in a similar way will be included in the present study.

We will search Google scholar, Medline/PubMed, Scopus, Embase, Web of Science, ProQuest, and Pedro databases from the time of inception of these databases to December 31, 2021. Based on the principle of comprehensiveness, in addition to databases, two key journals (Journal of Electromyography and Kinesiology, and Clinical Biomechanics) and reference lists related to previous related systematic reviews will be reviewed. Various combinations of search terms will be determined based on the MESH and Free text word techniques (see additional file 2).

\section{Study Selection:}

Identified articles will be managed using the Mendeley Reference Manager software version 1.19.5, where duplicate articles will be removed. A two-step screening process will be performed by two reviewers based on the title and abstract, followed by a full-text review. Two independent reviewers will screen the studies (categorization of initially identified articles, followed by initial screening based on the title and abstract). Potentially relevant articles will be evaluated against inclusion and exclusion criteria by both reviewers. In case of a disagreement, a consensus will be reached through discussion, or, if necessary, the third reviewer will be consulted. The entire process of study selection is shown in the PRISMA flow diagram (Fig1).

\section{Data collection process:}

The data will be extracted independently by two reviewers from each study. Disagreements will be resolved by a third reviewer. We will use a data extraction form that we have designed (See additional 
file3). Information extraction form includes description of the: study details (name of the first author, year of publication), description of study participants (gender, mean age, body mass index (BMI) and health status, duration of onset low back pain, sample size), method of studies (study design type, sampling technique, task and test procedure, number of trials), description of the motion capture system used (characteristics of motion analysis system, measurement frequency, kinematic outcome), statistical tests and the results of the studies. Any disagreement between the two reviewers in extracting the data of the studies included in the systematic study, will be resolved through a survey of the third reviewer.

\section{Risk of Bias and Methodological Quality:}

Eligible articles will be reviewed separately by two reviewers in terms of quality, and in cases where there is disagreement on the study score, paired reviewers will discuss disagreements to reach consensus, the third reviewer will evaluate as an arbitrator if consensus cannot be reached.

Risk of bias assessment will be done in the initial studies using the Newcastle-Ottawa Scale (NOS) tool, adapted versions [34]. The NOS tool contains eight items for cohort and case-control studies, and another scale has been adapted from the Newcastle-Ottawa Quality Assessment Scale for assessment of crosssectional studies. According to the type of study (cohort, case-control, and cross-sectional), all three versions of this tool contain items that are categorized in three sections: selection, comparability, and outcome (see additional file4) [35]. Researchers have been trained to use this quality assessment tool.

The results will be analyzed in the meta-analysis section from the perspective of the impact on the results of the study.

\section{Heterogeneity analysis:}

Q-Cochrane test will be used to show the heterogeneity of data in studies and statistical heterogeneity will be assessed using the $\mathrm{I}^{2}$ (inconsistency) statistic (range from 0 to $100 \%$ ) [36]. According to which, the $1^{2}$ index (inconsistency) is categorized as: 0-24.9 (mild heterogeneity) 25-49.9 (moderate heterogeneity) 50-74.9 (severe heterogeneity) 75-100 (highly severe heterogeneity) [37].

If substantial methodological heterogeneity exists, based on the conditions of the variable, subgroup analysis or meta-regression will be used to investigate the factors affecting heterogeneity. We will proceed to subgroup analysis by the type of kinematic parameter under investigation and type of performance studied and others.

Possible causes of heterogeneity include the type of function studied in the studies, the type of motion capture system, the examination of various segment of the body and different study design.

\section{Data Synthesis:}

We will perform a meta-analysis if the minimum criteria for achieving quantitative synthesis are met (i.e., lack of highly severe heterogeneity and the existence of at least four eligible studies) [38] .Depending on 
the methodological heterogeneity that will occur, fixed effect model (FEM) or random effect model (REM) will be administered [39]. The combination of data will be done in Forest Plot .Visually inspecting funnel plots will be used to evaluate the publication bias, which will be based on variables reported by at least ten studies [40]. In cases that the number of studies is less than ten, the publication bias will be examined using Begg or Egger statistical test methods [41]. Differences between quantitative kinematic parameters in people with NS-LBP and healthy individuals will be calculated as between-group standardized mean differences (SMD) (95\% confidence intervals (CI)). For categorical data, we will use odds ratios (OR) or risk ratios (RR) with 95\% confidence intervals using Stata software (version 14) [42]. If there is heterogeneity, we will perform subgroup analysis based on the type of performance (e.g., trunk flexion, sit to stand, etc.) and study design (cohort and case-control studies).

\section{Evaluation of the effect of methodological quality factor on the results:}

We will evaluate the relationship between the quality of studies and the effect size (ES) using subgroup analysis in the groups resulting from quality assessment. After quality assessment, which will be done on a score-based or categorical basis on included studies, we will divide the studies into at least three subgroups based on quality score or classification if possible, and using Stata software we will analyze subgroups in different quality subgroup. Then, based on the results, if there is a relationship between the quality of studies and the results, the final conclusion will be based on higher quality studies.

\section{Sensitivity analysis:}

We will use the one-out-remove method for the purpose of sensitivity analysis in Stata software using the metaninf module [43].

\section{Discussion}

To the best of our knowledge, this will be the first systematic review study to compare the difference in kinematic parameters of lumbo-pelvic and lower limb joints, specifically in the NS-LBP group, with healthy individuals. In the past, review studies have been performed comparing people with LBP with healthy people, regardless of the heterogeneity of the causes of LBP, or some studies have reviewed a kinematic factor only in one joint $[22,29]$.

The current systematic review will be a comprehensive investigation of the difference between the kinematic parameters of the lower limb joints and the lumbopelvic region between NS-LBP patients and healthy individuals during different functions.

Since the kinematic assessment of the lower extremities and pelvic joints during daily activities may be helpful in achieving the underlying factors causing LBP, a better understanding of kinematic changes in everyday functions is critical to improving treatment solutions, and the findings of our systematic review can be helpful for health care providers, researchers, and decision makers.

\section{Limitations}


Our research will not include studies on kinematic differences in gait performance, and only studies with full English text will be included; therefore, there are language limitations, and since we only include fulltext articles in the study, potential publication bias may be introduced.

\section{Abbreviations}

LBP: low back pain, CLBP: chronic low back pain, NS-LBP: non-specific-low back pain, ROM: range of motion, PRISMA-P: Preferred Reporting Items for Systematic Reviews and Meta-Analyses Protocol, AV: average velocity, MV: maximal velocity, L/H ratio: lumbar/hip motion ratios, BMI: body mass index, NOS: Newcastle - Ottawa Quality Assessment Scale, FEM: Fixed effect model, REM: Random effect model, Cl: Confidence interval[MD: Mean difference.SMD (standard Mean difference), OR: Odds ratio.

\section{Declarations}

\section{Ethics and dissemination}

Since the present study is a systematic review of the studies and no new data will be collected, there is no need to Ethical approval and patient consent.

\section{Acknowledgements}

Not applicable

\section{Funding}

This study did not receive funding

\section{Availability of data and materials}

Supporting data is attached in the Additional files

\section{Authors' contributions}

MN had the original idea for the systematic review.

FGh and AMA wrote the protocol. FGh and AMA were each contribute in the conception, design, and editing of the study. All authors read and approved the final manuscript.

\section{Ethics approval and consent to participate}

Not applicable 
Not applicable

\section{Competing interests}

The authors declare that they have no competing interests

\section{Authors' information}

${ }^{1}$ Department of Physical therapy, University of Social Welfare and Rehabilitation Sciences, Tehran, , University of North Georgia, Dahlonega, USA. ${ }^{2}$

\section{References}

1. Biglarian A, Seifi B, Bakhshi E, Mohammad K, Rahgozar M, Karimlou M, et al. Low back pain prevalence and associated factors in Iranian population: findings from the national health survey. Hindawi Publishing Corporation, Pain Research and Treatment 2012, doi:10.1155/2012/653060

2. Hoy D, Brooks P, Blyth F, Buchbinder R. The Epidemiology of low back pain. Best Pract Res Clin Rheumatol [Internet]. 2010,24(6):769-81.

3. Meucci RD, Fassa AG, Faria NMX \%J R de saude publica. Prevalence of chronic low back pain: systematic review. 2015,49:73.

4. Steffens D, Maher CG, Pereira LSM, Stevens ML, Oliveira VC, Chapple M, et al. Prevention of low back pain: a systematic review and meta-analysis. 2016,176(2):199-208.

5. Airaksinen O, Brox Jl, Cedraschi C, Hildebrandt J, Klaber-Moffett J, Kovacs F, et al. European guidelines for the management of chronic nonspecific low back pain. Eur Spine J 2006,15:S192-300

6. Costa L da CM, Maher CG, McAuley JH, Hancock MJ, Herbert RD, Refshauge KM, et al. Prognosis for patients with chronic low back pain: inception cohort study. Bmj. 2009,339:b3829.

7. Sadeghisani M, Manshadi FD, Kalantari KK, Rahimi A, Namnik N, Karimi MT, et al. Correlation between hip rotation range-of-motion impairment and low back pain. A literature review. 2015,17(5):455-62.

8. Kim M, Yi C, Kwon O, Cho S, Cynn H, Kim Y, et al. Comparison of lumbopelvic rhythm and flexionrelaxation response between 2 different low back pain subtypes. Spine (Phila Pa 1976). 2013 Jul,38(15):1260-7.

9. Zazulak BT, Hewett TE, Reeves NP, Goldberg B, Cholewicki J \%J TA journal of sports medicine. Deficits in neuromuscular control of the trunk predict knee injury risk: prospective biomechanicalepidemiologic study. 2007,35(7):1123-30.

10. Mitchell K, Porter M, Anderson L, Phillips C, Arceo G, Montz B, et al. Differences in lumbar spine and lower extremity kinematics in people with and without low back pain during a step-up task: a crosssectional study. 2017,18(1):369.

11. Murphy DR, Byfield D, McCarthy P, Humphreys K, Gregory AA, Rochon R \% J J of manipulative, et al. Interexaminer reliability of the hip extension test for suspected impaired motor control of the lumbar 
spine. 2006,29(5):374-7.

12. Sung PS. A compensation of angular displacements of the hip joints and lumbosacral spine between subjects with and without idiopathic low back pain during squatting. J Electromyogr Kinesiol. 2013,23(3):741-5.

13. Sutherlin MA, Hart JM \% J J of SR. Hip-abduction torque and muscle activation in people with low back pain. 2015,24(1):51-61.

14. Roach SM, San Juan JG, Suprak DN, Lyda M, Bies AJ, Boydston CR. Passive hip range of motion is reduced in active subjects with chronic low back pain compared to controls. Int J Sport Phys Ther. 2015/02/25. 2015,10(1):13-20.

15. Cooper NA, Scavo KM, Strickland KJ, Tipayamongkol N, Nicholson JD, Bewyer DC, et al. Prevalence of gluteus medius weakness in people with chronic low back pain compared to healthy controls. Eur Spine J. 2015/05/27. 2016,25(4):1258-65.

16. Burns SA, Mintken PE, Austin GP. Clinical decision making in a patient with secondary hip-spine syndrome. Physiother Theory Pract. 2011 Jul,27(5):384-97.

17. Van Dillen LR, Gombatto SP, Collins DR, Engsberg JR, Sahrmann SA. Symmetry of timing of hip and lumbopelvic rotation motion in 2 different subgroups of people with low back pain. Arch Phys Med Rehabil. 2007 Mar,88(3):351-60.

18. M. Sadeghisani, V. Sobhani, E. Kouchaki, A. Bayati, A. A. Ashari, and M. Mousavi, "Comparison of Lumbopelvic and Hip Movement Patterns During Passive Hip External Rotation in Two Groups of Low Back Pain Patients with and without Rotational Demand Activities.," Ortop. Traumatol. Rehabil., 2015, 17 (6): 611-618.

19. S. A. Scholtes, B. J. Norton, C. E. Lang, and L. R. Van Dillen, "The effect of within-session instruction on lumbopelvic motion during a lower limb movement in people with and people without low back pain.," Man. Ther., 2010, 15(5): 496-501.

20. H. J. Dananberg and M. Guiliano "Chronic low-back pain and its response to custom-made foot orthoses," J Am Podiatr Med Assoc., 1999 Mar,89(3):109-17.

21. G. L. K. Shum, J. Crosbie, and R. Y. W. Lee, "Effect of low back pain on the kinematics and joint coordination of the lumbar spine and hip during sit-to-stand and stand-to-sit," Spine (Phila. Pa. 1976)., vol. 30, no. 17 , 2005,30 (17):1994-2004.

22. Laird RA, Gilbert J, Kent P, Keating JL. Comparing lumbo-pelvic kinematics in people with and without back pain: a systematic review and meta-analysis. BMC Musculoskelet Disord. 2014Jul, 15 (): 15229.

23. Nolan D, O'Sullivan K, Newton C, Singh G, Smith BE. Are there differences in lifting technique between those with and without low back pain? A systematic review. Scand J pain. 2020,20(2):215-227.

24. Borkan, J., et al., Advances in the field of low back pain in primary care: a report from the fourth international forum. 2002. 27(5): p. E128-E132.

25. McCarthy, C.J., et al., The biopsychosocial classification of non-specific low back pain: a systematic review. Physical Therapy 2004. 9(1): p. 17-30. 
26. Leboeuf-Yde, C., J.M. Lauritsen, and T.J.S. Lauritzen, Why has the search for causes of low back pain largely been nonconclusive?spine. 1997.22 (8): p. 877-881

27. B. W. Koes, Mw. Van Tulder, and S. Thomas, “Diagnosis and treatment of low back pain,” Bmj, 2006. 332(7555), pp. 1430-1434

28. J. Liao et al., "Acupoint injection for nonspecific chronic low back pain: A protocol of systematic review," Medicine. 2019. 98(29)

29. Avman MA, Osmotherly PG, Snodgrass S, Rivett DA. Is there an association between hip range of motion and nonspecific low back pain? A systematic review. Musculoskelet Sci Pract. 2019,42():3851.

30. M. J. H. van Dijk et al., "Assessment instruments of movement quality in patients with non-specific low back pain: A systematic review and selection of instruments," Gait Posture, 2020,76():346-357

31. Page MJ, McKenzie JE, Bossuyt PM, et al. The PRISMA 2020 statement: an updated guideline for reporting systematic reviews. BMJ. 2021,372(71).

32. Gombatto, S. P., Brock, T., DeLork, A., Jones, G., Madden, E., \& Rinere, C. Lumbar spine kinematics during walking in people with and people without low back pain. Gait \& Posture, 2015,42(4), 539-44.

33. Airaksinen O, Brox Jl, Cedraschi C, et al. Chapter 4. European guidelines for the management of chronic nonspecific low back pain. Eur Spine J. 2006,15 suppl 2:S192-S300. https://doi. org/10.1007/s00586-006-1072-1

34. Wells GA, Shea B, O'Connell D, et al. The Newcastle-Ottawa Scale (NOS) for assessing the quality of nonrandomized studies in meta-analyses. Available at: http://www.ohri.ca/programs/clinical_epidemiology/oxford.asp. Accessed August 19, 2019.

35. Stang A. Critical evaluation of the Newcastle-Ottawa scale for the assessment of the quality of nonrandomized studies in meta-analyses. Eur J Epidemiol. 2010, 25:603-5.

36. Higgins J. P. T.,Thompson S. G., Deeks J. J., \& Altman D. G.Measuring inconsistency in meta-analysis.

British Medical Journal, 2003, 327: 557-560

37. Higgins JPT, Green S, editors. Cochrane Handbook for Systematic Reviews of Interventions Version 5.1.0 [updated March 2011]. The Cochrane Collaboration, 2011.. Accessed on August 2016

38. Higgins JPT, Thomas J, Chandler J, Cumpston M, Li T, Page MJ, et al. Cochrane handbook for systematic reviews of interventions. John Wiley \& Sons, 2019.

39. Schmidt FL, Oh I, Hayes TL. Fixed-versus random-effects models in meta-analysis: Model properties and an empirical comparison of differences in results. Br J Math Stat Psychol. 2009,62(1):97-128

40. Higgins JPT, Green S. Cochrane handbook for systematic reviews of interventions (version 5.1.0, March 2011). Available at: http://www.cochranehandbook.org/. Accessed 11 May 2020

41. Egger $M$, Smith GD, Schneider M, Minder C. Bias in meta-analysis detected by a simple, graphical test. Bmj. 1997,315(7109):629-34. 
42. Whitehead A, Whitehead J. A general parametric approach to the meta-analysis of randomized clinical trials. Statistics in medicine1991,10: 1665-77,

43. Sterne JAC, Sutton AJ, loannidis JPA, Terrin N, Jones DR, Lau J, et al. Recommendations for examining and interpreting funnel plot asymmetry in meta-analyses of randomised controlled trials. Bmj. 2011,343.

\section{Figures}

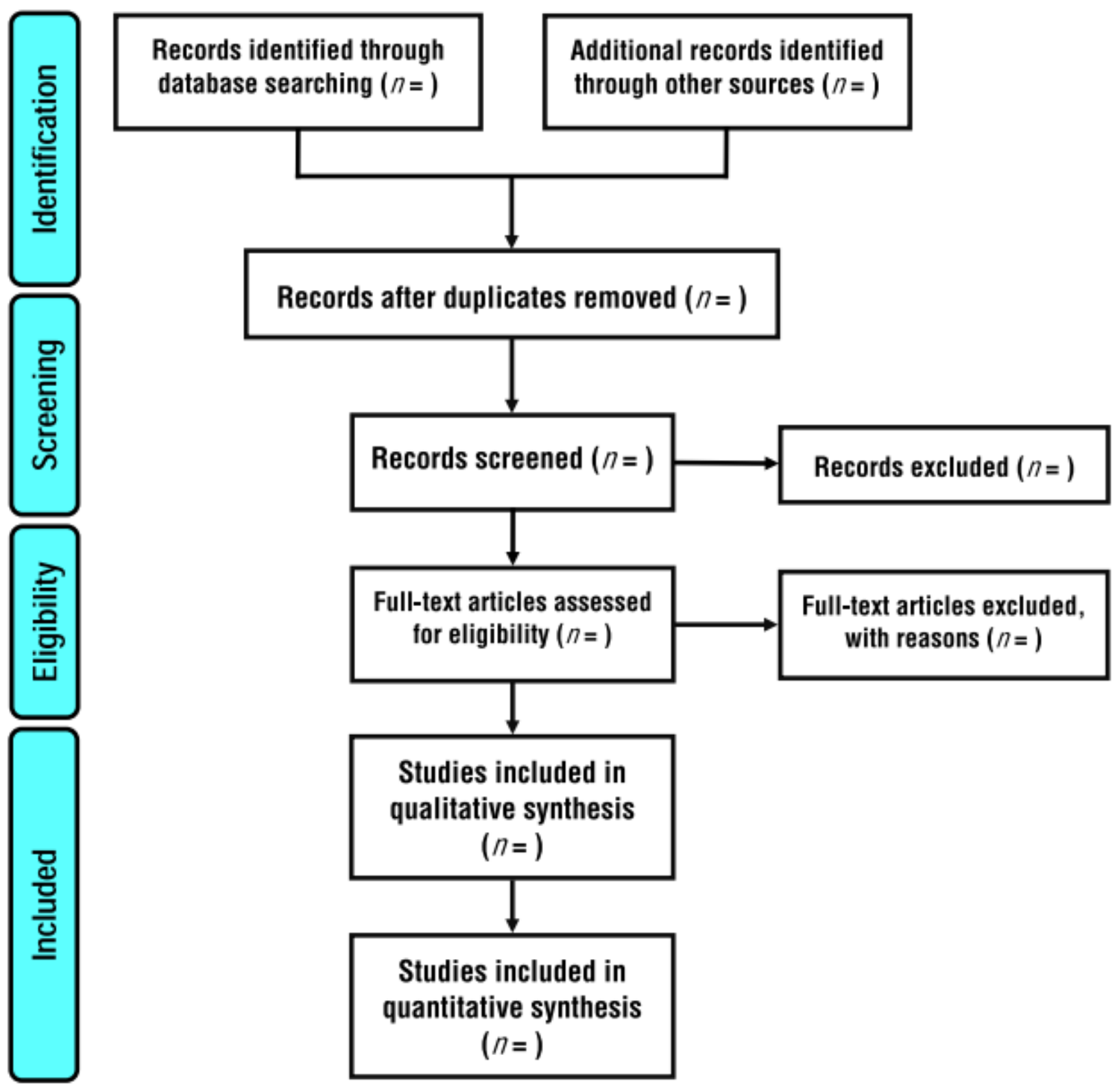

Figure 1

The entire process of study selection is shown in the PRISMA flow diagram

\section{Supplementary Files}


This is a list of supplementary files associated with this preprint. Click to download.

- PRISMAPchecklist.1.docx

- Additionalfile2.docx

- dataextractionform.3.docx

- riskosappraisalasessment.4.docx 